\title{
Korean Cultural Adaptation of WHODAS 2.0 (36-Item Version): Reliability and Linking to ICF
}

\author{
Ju Min Song, Hae Jung Lee \\ Department of Physical Therapy, College of Health and Welfare, Silla University, Busan, Korea
}

Purpose: This study was to conduct a Korean cultural adaptation of the WHO disability assessment schedule (WHODAS) 2.0: 36-item version.

Methods: An internationally standardized process of translation and cultural adaptation of an instrument was used to develop a Korean version of WHODAS 2.0: 36-item version. Linking each item into the International Classification of Functioning, Disability and Health (ICF) was also conducted in order to ensure the concepts in the translated instrument were compatible with ICF. All translated versions of the instrument, linking results and feedback from participants were used for the final adaptation of the Korean version of the instrument. The Korean version of the instrument was assessed twice on different occasions to examine Inter- and intra-rater reliability, and the intra-class correlation coefficient (ICC) was calculated.

Results: Twelve participants were involved in the translation and linking process. Ninety-five volunteers were invited to participate to examine the reliability of the instrument. Fifty participants completed the self-rated version of the instrument and 45 finished the interviewer version. The Korean WHODAS 2.0: 36-item version was found to have excellent reliability: self-rated version and interviewer version reliability coefficients were ICC $=0.92$ and ICC $=0.94$, respectively. Thirty-four items of the translated instrument were to be linked to ICF categories. Some adaptation was made; details and a familiar example were added to help respondents answer the questions.

Conclusion: The study results show that the adaptation of the 36-item version of WHODAS 2.0 to Korean was successful and the instrument is ready for use in testing its psychometric properties.

Keywords: WHODAS 2.0: 36 item version, Korean cultural adaptation, ICF

\section{INTRODUCTION}

Diagnosis alone is insufficient in explaining or understanding the overall status of a patient. ${ }^{1}$ Information on functioning is considered an important factor in overall health status, as it may describe, in general, how well a person is able to function in daily life areas.

When assessing functional status, it is common to use a self-report instrument in clinical practice and relevant research. ${ }^{2} \mathrm{~A}$ large number of generic and disease-specific instruments have been developed to measure the functional status of patients or persons with disabilities. Many are simple, reliable, and practical, making them suitable for quality assurance and research activities. Nevertheless, the majority had been developed before the introduction of the In-

Received Dec 4, 2018 Revised Dec 31, 2018

Accepted Dec 31, 2018

Corresponding author Hae Jung Lee

E-mail hjlee@silla.ac.kr ternational Classification of Functioning, Disability, and Health (ICF) biopsychosocial conceptual model and are less likely to contain a clear biopsychosocial concept. ${ }^{3.4}$ Therefore, most such instruments were limited in their inclusion of the biopsychosocial model or were focused on a very specific area, e.g. activity of daily living or hand function. ${ }^{5}$ Further, most instruments were developed for a Western cultural background and were in English.

ICF is a classification system for assessing health and disability within a biopsychosocial conceptual model, but the ICF is considered impractical for assessing and measuring disability in daily practice. ${ }^{6}$ WHODAS 2.0 was developed by WHO to address the above-mentioned problems and is based on the ICF concept of obtaining a general evaluation and measurement of health conditions

Copylight (C2018 The Korea Society of Physical Therapy

This is an Open Access article distribute under the terms of the Creative Commons Attribution Non-commercial License (Http:// creativecommons.org/license/by-nc/4.o.) which permits unrestricted non-commercial use, distribution, and reproduction in any medium, provided the original work is properly cited. 
and disabilities. WHODAS 2.0 also provides a standardized way to measure health and disability across cultures. WHODAS 2.0 has a unique feature, that is, it is directly linked to ICF, distinguishing it from other disability measures. ${ }^{7}$ Functioning measurement tools have been linked to ICF to promote communications among relevant professionals in Korea. ${ }^{5,8}$

WHODAS II was published in 2004 based on the WHO psychiatric disability assessment schedule which was designated to assess the extent of disability associated with a psychiatric condition. WHO also developed a second version (WHODAS 2.0) as a general measure of functioning and disability in major life domains. ${ }^{?}$

WHODAS 2.0 has been translated into many languages, including Portuguese, French, German, Norwegian, Italian, Russian, Japanese, Thai, and Chinese ${ }^{9-11}$ and has been validated in various settings and countries. WHODAS 2.0 can assess disability in a culturally sensitive way across a standard rating scale. However, a Korean version of the 36-item version of WHODAS 2.0, including self-rated and interviewer versions, is not yet available.

The purposes of this study were to develop a Korean version of the 36-item WHODAS 2.0 (K-WHODAS 2.0: 36-item) using a standardized process of translation and adaptation of instruments, to link each of the items in the Korean version to ICF and to test the reliability of $K$-WHODAS 2.0: 36-item.

\section{METHODS}

\section{Subjects}

\section{1) Cultural adaptation}

Twelve persons participated in the translating process. Eight of them had Korean as their mother tongue and translated the 36-item WHODAS 2.0 version into Korean. Two persons had English as their mother language and did backward translation of the Korean translated instrument into English. Two researchers, who were experts in clinical studies and outcome measures, participated in reviewing all translating results.

\section{2) Linking process}

Two health professionals were invited to participate in linking the K-WHODAS 2.0: 36-item instrument into the relevant ICF categories. Invited professionals for linking were experts in ICF usage and education in rehabilitation medicine, and they had experience in research involving clinical measurement tools and ICF.

\section{3) Reliability test}

Ninety-five volunteers were recruited for reliability test of the KWHODAS 2.0: 36-item instrument: 50 of them participated to examine the self-rated version and 45 volunteers to assess the interviewer version. To be included in the study, volunteers had to be over 18 years of age, and use Korean as their mother tongue. Volunteers were excluded if they were illiterate or were seeking treatment for personal health conditions during the study period. All participants provided informed consent prior to collecting data.

\section{Experimental methods \\ 1) Study procedure}

\section{(1) Cultural adaptation into Korean}

The procedure used in this study followed a guideline proposed by Beaton and colleagues ${ }^{12}$ to establish the Korean cultural adaptation of WHODAS 2.0: 36-item version. The procedure involved in the forward translation from English to Korean, and backward translation to verify the original meanings were preserved. A pre-final version was developed to integrate all translated versions of the instrument. ICF terminology was recommended for translating, and terms in instruments for functional measurement were to be consistent with ICF, which is considered a standardized classification for functioning and disability.

\section{(2) Linking to ICF}

An ICF linking rule was applied to see whether Korean version of the instrument could reflect the intended content or concept of functioning, and the approve items were linked to the Korean version of ICF. ${ }^{13}$ The linking process of the study was carried out according to the description provide in previous study. ${ }^{14}$

\section{(3) Reliability test}

Each subject completed the instrument twice on different occasions to determine test-retest reliability. The interval between occasions was 3 to 7 days depending on participant's availability. On completion of the second test, an interview was conducted to collect information on any misunderstanding of the intended meaning in each item and to examine the results for missing or multiple responses for each item. All collected information was adapted for final development. 


\section{2) WHODAS 2.0: 36-item version}

WHODAS 2.0, developed by WHO in 2010, is a generic measurement tool for assessing health and disability in clinical practice or at the population level. WHODAS 2.0 captures the level of functioning in six life domains during the preceding 30 days. WHODAS 2.0 examines person's cognition (understanding and communicating), mobility (moving and getting around), self-care (attending to one's hygiene, dressing, eating and staying alone), getting along (interacting with other people), life activities (activities related to the household, work or study) and participation (joining in community activities, participating in society). There are seven types of WHODAS 2.0, including: Self-administration (36 items or 12 items), Interviewer (36, $12+24$, or 12 items), and Proxy (36 items or 12 items) instruments. ${ }^{15}$

A respondent should be informed of a couple of concepts before testing: degree of difficulty was to be interpreted as increased effort, discomfort or pain, slowness, or changes in the way the person does the activity; health condition was meant to include diseases, illnesses, injuries, mental or emotional problems, problems with alcohol, or problems with drugs. Averaging was done for good and bad days for the last 30 days; if an item was not experienced in the past 30 days, it was to be rated as N/A. The respondent's usual activity status should be answered with or without assistance.

Respondents scored each item on a 5-point Likert scale from 1 (no difficulty) to 5 (extreme or cannot do). Simple scoring is sum of scores of each assigned item. Complex scoring involved calculating scores for each domain with an overall disability score ranging from 0 (no disability) to 100 (full disability). Simple scoring could be used for a specific population in a clinical setting, whereas complexing scoring would be used for across population comparisons. Previously, it was reported that WHODAS 2.0 is reliable and applicable across cultures in all adult populations. ${ }^{11}$

\section{Data Analysis}

The demographic characteristics and simple scores from the WHODAS 2.0: 36-item self-rated version was analyzed using descriptive statistics. Intra-class correlation coefficients (ICC) were calculated for the K-WHODAS 2.0: 36-item instrument across two measurement occasions to examine the reliability of the instrument. All analyses in the study were performed using IBM SPSS version 24.

\section{RESULTS}

\section{Characteristics of subjects}

To assess the K-WHODAS 2.0: 36-item instrument's reliability, 95 subjects participated in the study. Fifty subjects (22 males), aged from 20 to 74 years (mean $40.82 \pm 16.10$ years), completed the selfrated version of the instrument and 45 (24 males), aged from 18 to 28 years (mean $22.11 \pm 2.45$ years) were interviewed to complete the interviewer version. The mean of simple score in the self-rated version was 61.04 (SD 53.05).

\section{K-version of WHODAS 2.0: 36-item instrument and linking the items to K-ICF categories}

ICF terminology was applied so that the translated instrument would be linked to ICF and also be understood easily by general Korean population. A few adaptations were made based on the results of the translations, links to ICF and information from interviews. The Western concept of sitting down was clarified as either sitting in a chair or sitting on the floor, which is common in the Korean culture. Different words with similar meaning within a sentence were changed to a single word to create a more natural question format. Familiar examples were added to help respondents understand an item easily. Household activity was considered to be more predominantly a women's role in Korea and it was replaced with the term 'daily routine activity' as it contained a broader meaning than household activity and reflected the meaning of the related items more appropriately (Appendix 1).

Thirty-four of the 36 items in the K-WHODAS 2.0 instrument were linked to 3 ICF chapters including self-care (D5), domestic life (D6) and community, social and civic life (D9), as well as 35 ICF categories. Thirty-five ICF categories included 5 in the body function domain and 30 the activity and participation domain (Table 1). The concepts related to two items related to health condition (D6.4, D6.7) were not able to be linked to appropriated ICF categories.

\section{Reliability}

The self-rated and interviewer versions of K-WHODAS 2.0: 36-item instrument had high reliability in the repeated measure assessment. The ICC values of each domain of the translated instrument were examined and are reported in Table 2. 
Table 1. Linking of items in the 36-item version of WHODAS 2.0 to ICF categories: concepts included in each WHODAS 2.0 item were compared and linked to the appropriate ICF category

\begin{tabular}{|c|c|c|}
\hline WHODAS 2.0 & Concept & ICF Category \\
\hline \multicolumn{3}{|l|}{ Domain 1: Understanding and communicating } \\
\hline D1.1 Concentrating on doing something for ten minutes & - Concentrating & $\begin{array}{l}\text { - b140 attention function, } \\
\text { d160 focusing attention }\end{array}$ \\
\hline D1.2 Remember to do important things & - Remembering & - b144 memory function \\
\hline D1.3 Analyzing and finding solutions to problems in day-to-day life & - Problem solving in day-to-day & $\begin{array}{l}\text { - b1646 Problem solving, } \\
\text { d175 Solving problems }\end{array}$ \\
\hline D1.4 Learning a new task, for example, learning how to get to a new place & - Learning a new task & • d155 Acquiring skills \\
\hline D1.5 Generally understanding what people say & - Understanding of spoken language & $\begin{array}{l}\text { - b152 Communicating with- } \\
\text { receiving-spoken messages }\end{array}$ \\
\hline D1.6 Starting and maintaining a conversation & $\begin{array}{l}\text { - Starting and maintaining a } \\
\text { conversation }\end{array}$ & $\begin{array}{l}\text { - d3500 Starting a conversation, } \\
\text { d3501 Sustaining a conversation }\end{array}$ \\
\hline \multicolumn{3}{|l|}{ Domain 2: Getting around } \\
\hline D2.1 Standing for long periods such as 30 minutes & - Standing for a long period & - d4154 Maintaining a standing position \\
\hline D2.2 Standing up from sitting down & - Standing up & • d4104 Standing \\
\hline D2.3 Moving around inside your home & - Moving around within one's house & - d4600 Moving around within the home \\
\hline D2.4 Getting out of your home & - Getting out from one's House & - d4602 Moving around outside the home \\
\hline D2.5 Walking a long distance such as a kilometre [or equivalent] & - Walking a long distance & • d4501 Walking long distances \\
\hline \multicolumn{3}{|l|}{ Domain 3: Self-care } \\
\hline D3.1 Washing your whole body & -Washing whole body & - d5101 Washing whole body \\
\hline D3.2 Getting dressed & - Getting dressed & - d540 Dressing \\
\hline D3.3 Eating & - Eating & • d550 Eating \\
\hline D3.4 Staying by yourself for a few days & - Staying by oneself for a few days & $\begin{array}{l}\text { • d850 Remunerative employment, } \\
\text { d820 School education, } \\
\text { d825 Vocational training, } \\
\text { d830 Higher education, } \\
\text { d8451 maintaining a job }\end{array}$ \\
\hline \multicolumn{3}{|l|}{ Domain 4: Getting along with people } \\
\hline D4.1 Dealing with people you do not know & - Dealing with people/stranger & - d730 Relating with stranger \\
\hline D4.2 Maintaining a friendship & - Maintaining friendship & - d7500 Informal relationships with friends \\
\hline D4.3 Getting along with people who are close to you & - Getting along with are close persons & $\begin{array}{l}\text { - d760 Family relationships, } \\
\text { d770 Intimate relationships }\end{array}$ \\
\hline D4.4 Making new friends & - Making a friend & - d7500 Informal relationships with friends \\
\hline D4.5 Sexual activities & - Sexual activities & - d7702 Sexual relationship \\
\hline \multicolumn{3}{|l|}{ Domain 5: Life activities } \\
\hline D5.1 Taking care of your household responsibilities & - Taking care of household & - d6 Domestic life \\
\hline D5.2 Doing most important household tasks well & - Doing important household & \\
\hline D5.3 Getting all the household work done that you needed to do & - Getting all household done & \\
\hline D5.4 Getting your household work done as quickly as needed & - Getting a household & \\
\hline D5.5 Your day-to-day work/school & - Day-to-day work/school & $\begin{array}{l}\text { - d850 Remunerative employment, } \\
\text { d820 School education, } \\
\text { d825 Vocational training, } \\
\text { d830 Higher education, } \\
\text { d8451 maintaining a job }\end{array}$ \\
\hline D5.6 Doing your most important work/school tasks well & - Doing work/school task & \\
\hline D5.7 Getting all the work done that you need to do & - Getting all the work done & \\
\hline D5.8 Getting your work done as quickly as needed & - Getting work done & \\
\hline \multicolumn{3}{|l|}{ Domain 6: Participation in society } \\
\hline $\begin{array}{l}\text { D6.1 How much of a problem did you have in joining in community activities (for example, } \\
\text { festivities, religious or other activities) in the same way as anyone else can }\end{array}$ & $\begin{array}{l}\text { - Community activities } \\
\text { (festivities, religious or other activities) }\end{array}$ & - d910 Community life \\
\hline $\begin{array}{l}\text { D6.2 How much of a problem did you have because of barriers or hindrances in } \\
\text { the world around you? }\end{array}$ & - World around you & - d9 Community, social and civic life \\
\hline $\begin{array}{l}\text { D6.3 How much of a problem did you have living with dignity because of the attitudes } \\
\text { and actions of others? }\end{array}$ & - Living with dignity & - d940 Human rights \\
\hline D6.4 How much time did you spend on your health condition, or its consequences? & • Health condition & $\mathrm{N} / \mathrm{A}$ \\
\hline D6.5 How much have you been emotionally affected by your health condition? & • Emotion & • b152 Emotional function \\
\hline D6.6 How much has your health been a drain on the financial resources of you or your family? & • Financial resources & - d8700 Personal economic resources \\
\hline D6.7 How much of a problem did your family have because of your health problems? & • Family's problem & N/A \\
\hline D6.8 How much of a problem did you have in doing things by yourself for relaxation or pleasure? & - Recreation or leisure & - d920 Recreation and leisure \\
\hline
\end{tabular}


Table 2. Test-retest reliability of the Korean version of WHODAS 2.0: 36 items. Intra-rater reliability was examined for the self-rated version and inter-rater reliability was assessed for the interviewer version

\begin{tabular}{llll}
\hline Measurement tools & \multicolumn{1}{c}{ Domain } & ICC & $95 \%$ Cl \\
\hline WHODAS 2.0: 36-item self-rated version & D1: Cognition & 0.89 & $0.82-0.93$ \\
& D2: Mobility & 0.76 & $0.61-0.86$ \\
& D3: Self-care & $0.76-0.92$ & $0.85-0.95$ \\
& D4: Getting along & 0.91 & $0.77-0.92$ \\
WHODAS 2.0: 36-item interviewer version & D5: Life activities & 0.86 & $0.85-0.95$ \\
& D6: Participation & 0.91 & $0.86-0.95$ \\
& Total & 0.92 & $0.85-0.97$ \\
& D1: Cognition & 0.94 & $0.67-0.90$ \\
& D2: Mobility & 0.82 & 1 \\
& D3: Self-care & 1 & $0.90-0.97$ \\
& D4: Getting along & 0.95 & $0.80-0.95$ \\
& D5: Life activities & 0.90 & $0.72-0.92$ \\
& D6: Participation & 0.86 & $0.91-0.96$ \\
\hline
\end{tabular}

\section{DISCUSSION}

This study was conducted to develop a Korean version of the 36item WHODAS 2.0 instrument following a standardized process of translation and adaptation of instrument. The developed K-WHODAS 2.0: 36-item instrument is considered easy to understand and use. Each item of the instrument was linked to the Korean ICF version. Reliability of the instrument was observed to be excellent.

Adaptations were made based on the translation and linking to ICF results. Stand up from sitting was commonly understood for Korean culture as standing up from sitting on a chair or on the floor. This concept of standing up from sitting down (item D2.2) was different from original WHODAS 2.0 manual in which sitting on the floor was excluded. ${ }^{15}$ Therefore this concept needed to be expressed in the Korean version of the instrument and an example was added to explain 'standing up from sitting in a chair'. In the effect of difficulties section, 'cut back' and 'reduce' was translated as 'reduce' for both terms because the context was more natural and it did not change the concept.

The concepts of 'staying by yourself for a few days' (D3.4) included whether the subject could spend a few days within their home environment, i.e. the current residence on doing daily activities and talking care of household, and this item was linked to self-care (d5), acquisition of goods and services (d620), household tasks (d630d649) and caring for household objects (d650). With the exception of acquisition of a place to live (d610), inclusions in this concept are consistent with those in a previous study?

There were examples of items that should be added to make a respondent understand the item easier. For example, learning a new task (D1.4) includes learning how to get a new place. Leaning a new task could depend on the respondents' cognition level such that getting a new place could be considered to be difficult to understand. Simpler and a broader range of examples should be added, such as learning how to make a call using a smartphone.

Household in life activities' domain (D5.1-4) has been commonly considered to be a woman's role in Korean culture. Most elderly male participants had limited responses in those items, with or without health condition. It was recommended that household tasks in this domain should be included and expressed for every member within the house, for example, managing finances, car and home repairs for males within a family in Korea. Therefore, 'daily routine activities', which could include various roles within the household, was chosen as the title of this domain in the K-WHODAS 2.0: 36-item instrument. This concept adaptation was also applied in a previous study. ${ }^{16}$

Work or school activities were also part of the life activities domain and related items were linked to ICF categories including maintaining a job (d8451), remunerative employment (d850), school education (d820), vocational training (d825), higher education (d830). These results were consistent with a previous study, with the exception of maintaining a job (d8451)?

It was observed that the definitions for acquiring, keeping, and 
terminating a job (d845) and remunerative employment (d850) in ICF concepts were very similar with "seeking, finding and choosing employment, being hired and accepting employment, maintaining..." and "... seeking employment and getting a job, doing the required tasks of the job, ......, respectively. It was confusing to choose related categories to explain this item within the ICF concept. It is recommended that those ICF categories be updated to clarify the included concept and the extent of the categories.

Health condition-related items (D6.4, D6.7) were not linked to ICF categories. Even though all the items in the instrument were related to health condition, they included specific activities. The items in D6.4 and D6.7 asked specific questions about health conditions and problems of family members' experiencing, respectively?

Excellent inter-rater reliability was observed in this study. This result is consistent with those in previous studies. ${ }^{910}$ Participants completing the interviewer version of the instrument provided very similar general characteristics; similar in age, education and health condition. It is suggested that a broader subjects characteristics, such as that in a clinical population, should be invited to participate in further studies. In conclusion, the results suggest that the K-WHODAS 2.0: 36-item instrument was successfully developed and the instrument is deemed ready for testing its psychometric properties.

\section{REFERENCES}

1. Prodinger B, Tennant A, Stucki G. Standardized reporting of functioning information on ICF-based common metrics. Eur J Phys Rehabil Med. 2018;51(1):110-7.

2. Worth A, Hammersley V, Knibb R et al. Patient-reported outcome measures for asthma: a systematic review. NPJ Prim Care Respir Med. 2014; 24:14020.

3. Geyh S, Cieza A, Kollerits B et al. Content comparison of health-related quality of life measures used in stroke based on the international classifi- cation of functioning, disability and health (ICF): a systematic review. Qual Life Res. 2007;16(5):833-51.

4. Wang P, Zhang J, Liao W et al. Content comparison of questionnaires and scales used in low back pain based on the international classification of functioning, disability and health: a systematic review. Disabil Rehabil. 2012;34(14):1167-77.

5. Song J, Lee H. Mapping items of functioning questionnaires into the international classification of functioning, disability and health: stroke. J Kor Phys Ther. 2016;28(5):341-7.

6. Garin O, Ayuso-Mateos JL, Almansa J et al. Validation of the "World Health Organization Disability Assessment Schedule, WHODAS-2" in patients with chronic diseases. Health Qual Life Outcomes. 2010;8:51.

7. Üstün TB, Chatterji S, Kostanjsek N et al. Development the world health organization disability assessment schedule 2.0. Bull World Health Organ. 2010;88(11):815-23.

8. Lee $\mathrm{H}$, Song J. Mapping items of functioning questionnaires into the international classification of functioning, disability and health: low back pain. J Kor Phys Ther. 2016;28(5):321-7.

9. Chiu TY, Yen CF, Chou CH et al. Development of traditional Chinese version of world health organization disability assessment schedule 2.0 36 - item (WHODAS 2.0) in Taiwan: validity and reliability analyses. Res Dev Disabil. 2014;35(11):2812-20.

10. Silva C, Coleta I, SilvaI AG et al. Adaptation and validation of whodas 2.0 in patients with musculoskeletal pain. Rev Saúde Pública. 2018;47(4):16.

11. Federici S, Bracalenti M, Meloni F et al. World health organization disability assessment schedule 2.0: an international systematic review. Disabil Rehabil. 2017;39(23):2347-80.

12. Beaton DE, Bombardier C, Guillemin F et al. Guidelines for the process of cross-cultural adaptation of self-report measures. Spine. 2000;25(24): 3186-91.

13. WHO. International classification of functioning, disability, and health: Korean version. Seoul, Ministry of Health and Welfare, 2016.

14. Cieza A, Geyh S, Chatterji S et al. ICF linking rules: an update based on lessons learned. J Rehabil Med. 2005;37(4):212-8.

15. WHO. Measuring health and disability: manual for WHO disability assessment schedule (WHODAS 2.0). Malta, World Health Orgnization, 2010.

16. Lee H, Song J. The Korean language version of stroke impact scale 3.0: cross-cultural adaptation and translation. J Korean Soc Phys Med. 2015;10(3):47-55. 